\title{
A Self-Oscillating System to Measure the Conductivity and the Permittivity of Liquids within a Single Triangular Signal
}

\author{
Sylvain Druart, Denis Flandre, and Laurent A. Francis \\ ICTEAM Institute, Université catholique de Louvain, Place du Levant 3, 1348 Louvain-la-Neuve, Belgium \\ Correspondence should be addressed to Laurent A. Francis; laurent.francis@uclouvain.be
}

Received 11 April 2013; Accepted 12 December 2013; Published 13 February 2014

Academic Editor: Jiri Homola

Copyright (C) 2014 Sylvain Druart et al. This is an open access article distributed under the Creative Commons Attribution License, which permits unrestricted use, distribution, and reproduction in any medium, provided the original work is properly cited.

\begin{abstract}
We present a methodology and a circuit to extract liquid resistance and capacitance simultaneously from the same output signal using interdigitated sensing electrodes. The principle consists in the generation of a current square wave and its application to the sensor to create a triangular output voltage which contains both the conductivity and permittivity parameters in a single periodic segment. This concept extends the Triangular Waveform Voltage (TWV) signal generation technique and is implemented by a system which consists in a closed-loop current-controlled oscillator and only requires DC power to operate. The system interface is portable and only a small number of electrical components are used to generate the expected signal. Conductivities of saline $\mathrm{NaCl}$ and $\mathrm{KCl}$ solutions, being first calibrated by commercial equipment, are characterized by a system prototype. The results show excellent linearity and prove the repeatability of the measurements. Experiments on water-glycerol mixtures validate the proposed sensing approach to measure the permittivity and the conductivity simultaneously. We discussed and identified the sources of measurement errors as circuit parasitic capacitances, switching clock feedthrough, charge injection, bandwidth, and control-current quality.
\end{abstract}

\section{Introduction}

The quantification and qualification of liquid properties such as ionic concentration, permittivity, or $\mathrm{pH}$ are of major interest for the fluid identification in several domains like medicine, biology, or chemistry. In order to face this complex challenge, instrumentation solutions are constantly developed. This paper focuses on electrical measurements of liquid mixtures in order to extract the impedance modeled as an $R-C$ parallel dipole [1-3].

Classic sensing device used in most of these achievements is a solid-state interdigitated electrodes (IDE) sensor which is stimulated by a sinusoidal wave. The results in terms of fluid impedance are extracted out of the modulus and the phase of the response. A more effective way to stimulate the sensor is the use of a triangular signal (Triangular Waveform Voltage (TWV)) as described in [4] and applied to DNA hybridization detection [5]. In this paper, the proposed sensor interface implements the principle of a triangular wave voltage generation by a square current signal. This technique has been used successfully to sense constant phase element impedances but the extraction of the sensed data required the counting of all the elapsed periods of the triangular signal after its generation [6]. The present work uses this technique for measuring the conductivity and permittivity of liquids modeled as $R-C$ dipole. The first amelioration consists in isolating each voltage segment into a compact equation independent of the previous states of the oscillating wave. Therefore, the signal analysis is highly simplified and only requires sampling the data of a signal period to get all the expected parameters of a tested liquid. Secondly, our method implements a current-controlled oscillator (CCO) circuit of simple architecture so that the resulting solution requires a single DC source to operate. Autonomous systems are indeed highly advantageous to improve the compactness and the portability of sensing systems [7].

This paper first describes the IDE liquid sensor structure and defines its equivalent electric impedance; a finite element model (FEM) is used to compute the sensor electrical potential profile. The device stimulation theory is then demonstrated, resulting in a formal equation of a wave segment. The architecture of the CCO that implements the technique 


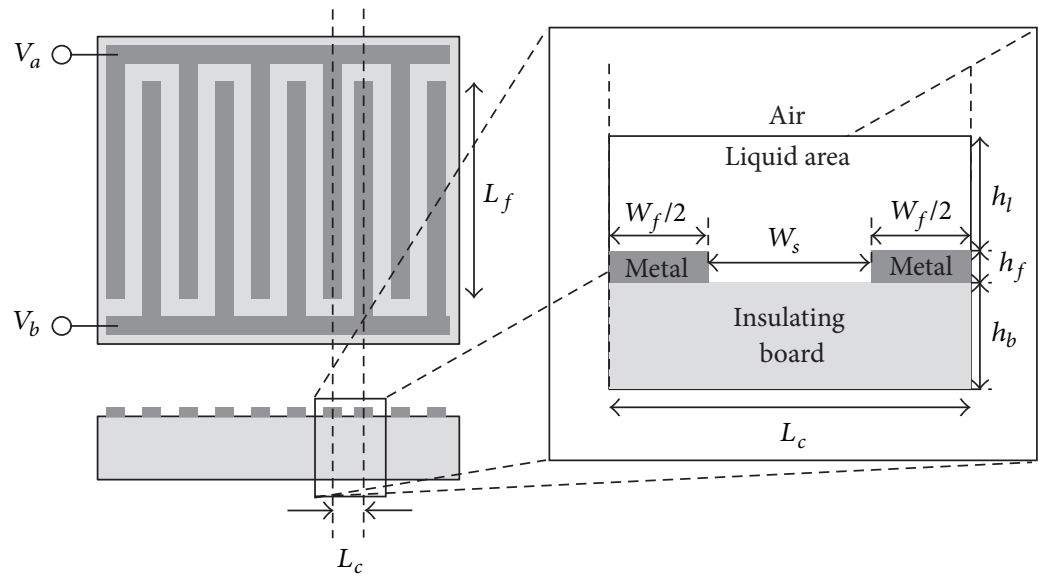

FIGURE 1: Global view and cross-section of the interdigitated electrodes (IDE).

is chosen and fabricated using printed circuit board (PCB) technology. Solutions of $\mathrm{NaCl}$ and $\mathrm{KCl}$ are first measured to test the system performances for conductivity sensing. Mixtures of water and glycerol are finally prepared to show the ability of the system to sense both the conductivity and permittivity measurement. The last part of the paper highlights the error sources in terms of circuit parasitic capacitances, bandwidth, and quality of the current source.

\section{Sensitive Element Model}

This first section defines the sensor equivalent model in terms of electrical components and highlights the sensed parameters. The device which is in contact with the fluid under test consists of an interdigitated electrodes (IDE) structure whose metal patterns are deposited on an insulating substrate. In order to establish the sensitive element model, we will consider the fluidic mixture as a material with a finite conductivity and a given relative permittivity.

2.1. Interdigitated Sensor Structure. The structure view is illustrated in Figure 1 which depicts on the left the device top and cross-views and on the right the periodic cell structure cross-section; the symbols $V_{a}$ and $V_{b}$ represent the device electric contacts. A cell is defined as the periodic element of an IDE structure. The calculation domain of the impedance is therefore bounded on a single cell structure while supposing the number of fingers is high enough to neglect the edge effects. All the geometrical parameters are reported in Table 1.

The electric model of the structure is given in Figure 2 and its impedance by (1). It consists of a global $R-C$ parallel dipole, with the components $R_{L}$ and $C_{L}$ being, respectively, linked to the fluid conductivity $\sigma_{L}$ and permittivity $\varepsilon_{L}$ by the geometrical factor defined in (2) as the cell constant $c_{k}$ expressed in $\mathrm{m}^{-1}$ [1]. The component $C_{B}$ is the parasitic substrate capacitance. In the present context, we suppose the use of techniques and materials that allow for neglecting the interface solid-liquid effects. The electrodes are made of gold, a highly cathodic material. Due to this property, the ionic adsorption on gold is low, thus lowering the faradaic
TABle 1: Parameter description.

\begin{tabular}{lcc}
\hline Name & Symbol & Units \\
\hline Tested fluid thickness & $h_{l}$ & $(\mathrm{~m})$ \\
Board thickness & $h_{b}$ & $(\mathrm{~m})$ \\
Electrodes height & $h_{f}$ & $(\mathrm{~m})$ \\
Electrodes width & $W_{f}$ & $(\mathrm{~m})$ \\
Electrodes spacing & $W_{s}$ & $(\mathrm{~m})$ \\
Cell width & $L_{c}$ & $(\mathrm{~m})$ \\
Electrodes length & $L_{f}$ & $(\mathrm{~m})$ \\
Electrodes number & $N_{f}$ & $(-)$ \\
\hline
\end{tabular}

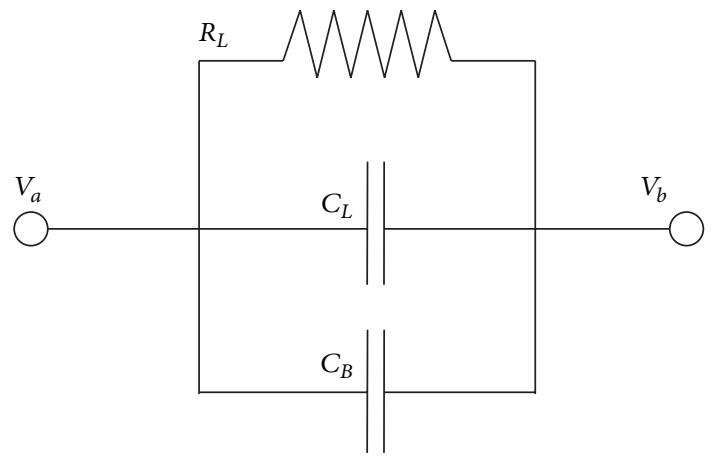

FIGURE 2: IDE electrical equivalent model.

current transfer [2] and the electrical double layer (EDL) formation [1]. The fingers are also considered wide enough in comparison with the typical thickness of the EDL to allow for neglecting the impact of double layer capacitance (i.e., $>1 \mu \mathrm{m}$ ) [8]:

$$
\begin{gathered}
Z_{S}=\frac{R_{L}}{1+s R_{L}\left(C_{L}+C_{B}\right)}, \\
c_{k}=\sigma_{L} R_{L}=\frac{\varepsilon_{L}}{C_{L}} .
\end{gathered}
$$

2.2. Finite Element Modeling. The impedance values are computed by finite elements modeling (FEM) with the use of COMSOL Multiphysics software. The time-harmonic Laplace 


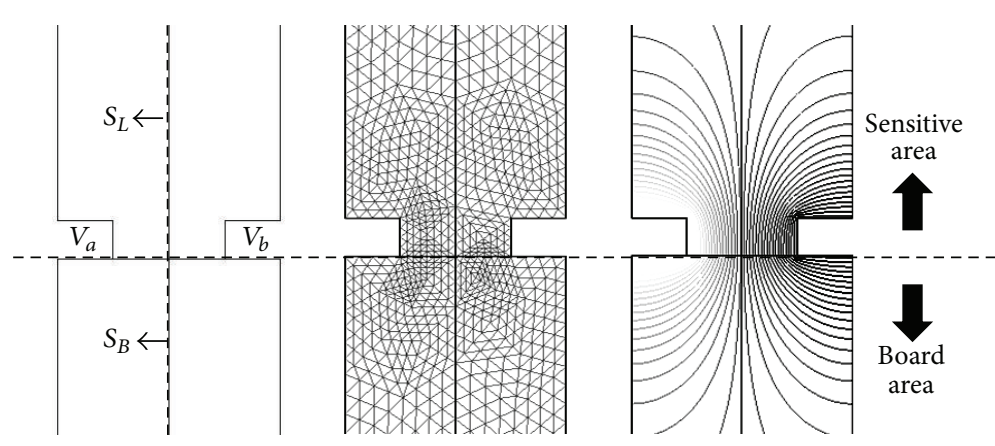

(a)

(b)

(c)

FIGURE 3: IDE cell cross-section: solving domain (a), mesh (b), and isopotential lines (c).
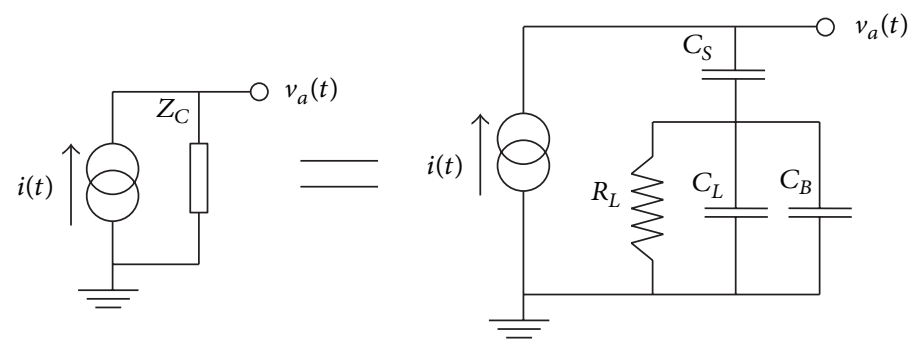

Figure 4: Impedance $Z_{C}$ stimulated by a current.

equation (3) is solved over a meshed domain bounded by a cell structure periodic element as shown in Figures 3(a) and 3(b). The global electric potential profile (Figure 3(c)) obtained after Laplace equation solution computation gives access to the electric field $\mathbf{E}$ present between the metal fingers. The integration of the electric field over the straight boundaries $S_{L}$ and $S_{B}$ shown in Figure 3(a) gives access to both cell constants in the bulk and in the sensitive area, $c_{k B}$ and $c_{k L}$ (4). The bulk and fluid capacitances and the fluid resistance are given by (5). The height of the resolution domain is set to be twice the cell width to enclose $99 \%$ of the electric energy [9]. FEM has been chosen because the IDE classical analytical formulas given in [9] do not consider the finger height and make the cell constant estimation less accurate:

$$
\begin{gathered}
-\nabla \cdot\left(\sigma+j \omega \varepsilon_{0} \varepsilon_{r}\right) \nabla V=0, \\
c_{k L}=\frac{\Delta V}{\int_{0}^{h_{l}} E_{x} d y}, \quad c_{k B}=\frac{\Delta V}{\int_{-h_{b}}^{0} E_{x} d y}, \\
R_{L}=\frac{c_{k L}}{\sigma_{L} N_{f} L_{f}}, \quad C_{L}=\frac{\varepsilon_{L} N_{f} L_{f}}{c_{k L}}, \quad C_{B}=\frac{\varepsilon_{B} N_{f} L_{f}}{c_{k B}} .
\end{gathered}
$$

a compact mathematical expression of the output signal that contains the fluid parameters.

3.1. Impedance Transient Analysis. The IDE structure model developed in the previous section is included in an electrical circuit with an additional serial capacitance $C_{S}$ as shown in Figure 4. The insertion of this capacitance is necessary to generate the triangular shape of the output signal; this cuts the DC path from the analog voltage node to the ground. The circuit loads the device under test with a square current signal $i(t)$ and the response voltage $v_{a}(t)$ is then probed. The total impedance $Z_{C}$ loaded by the square current is given by

$$
Z_{C}=\frac{1}{s C_{L}}+Z_{S}
$$

Applying the TWV theory in the case of the impedance $Z_{C}(s)$, a square-shaped current wave (7) of amplitude $I_{0}$ and period $2 T$ stimulates the impedance, with $u(t)$ being the unit hardstep function. As shown in [6], the two first steps of the current wave have, respectively, $I_{0} / 2$ and $3 I_{0} / 2$ of amplitude to keep mean value of the voltage response at zero:

\section{Sensor Stimulation Theory}

The theory exposed in this section explains how the sensitive element is stimulated. The sensing principle is based on the Triangular Waveform Voltage technique (TWV) as described in [4]. The goal of the proposed demonstration is to provide

$$
\begin{aligned}
i(t)= & \frac{I_{0}}{2} u(t)-\frac{3 I_{0}}{2} u(t-T) \\
& +2 I_{0} \sum_{n=2}^{\infty}(-1)^{n} u(t-n T) .
\end{aligned}
$$


The Laplace transform of the current wave is expressed by

$$
\begin{aligned}
I(s)= & \frac{I_{0}}{2 s}-\frac{3 I_{0} e^{-T s}}{2 s} \\
& +2 I_{0} \sum_{n=2}^{\infty}(-1)^{n} \frac{e^{-n T s}}{s} .
\end{aligned}
$$

The application of the inverse Laplace transform on the $Z_{C}(s) I(s)$ product gives the time domain voltage response wave expression given by (9), introducing three shaping parameters named in Table 2 as $\beta, \zeta$, and $\tau$. The plots sketched in Figure 5 illustrate the time progression of the current wave and the voltage output wave for several values of the model electrical components:

$$
\begin{aligned}
v_{a}(t)= & \frac{1}{2} u(t) \cdot\left[\beta t+\zeta\left(1-\zeta e^{(-t / \tau)}\right)\right] \\
& -\frac{3}{2} u(t-T) \cdot\left[\beta(t-T)+\zeta\left(1-e^{(-(t-T) / \tau)}\right)\right] \\
& +2 \beta \sum_{n=2}^{\infty}\left[(-1)^{n}(t-n T) \cdot u(t-n T)\right] \\
& +2 \zeta \sum_{n=2}^{\infty}\left[(-1)^{n} \cdot u(t-n T)\right] \\
& -2 \zeta \sum_{n=2}^{\infty}\left[(-1)^{n} e^{(-(t-T) / \tau)} \cdot u(t-n T)\right] .
\end{aligned}
$$

3.2. Response Wave Segmentation. The transient development gave the output voltage wave as a series expression. Each rising or falling wave segment contains all the information we are seeking about the tested impedance. A compact equation of wave segments thus needs to be calculated to extract the curve parameters $\beta, \zeta$, and $\tau$. The demonstration reported in the appendix derives the mathematical expression (10) of wave segments. The curve plotted in Figure 6 summarizes the parametric representation of a wave segment with the definition of a variable $\zeta^{\prime}$ (11). The segment wave comprises two distinct parts, an exponential one and a linear one. Their separation time point depends on the impedance parameters values:

$$
\begin{gathered}
v_{u}(t)=\beta t+\frac{2 \zeta}{1+e^{(-T / \tau)}}\left(1-e^{(-t / \tau)}\right), \\
\zeta^{\prime}=\frac{2 \zeta}{1+e^{(-T / \tau)}} .
\end{gathered}
$$

3.3. Model Interpretation. Equation (10) is the compact mathematical form of each wave segment. Three particular cases can be observed from this expression.

3.3.1. The Exponential Part of $v_{u}(t)$ Is Predominant. This first case appears when the time constant $\tau$ is greater than the current wave oscillation half period $(\tau \gg T)$. Figure $7(\mathrm{a})$ illustrates how this first case is reached by increasing the liquid resistance $R_{L}$ : the exponential part is dominant and
TABLE 2: Voltage response shaping parameters.

\begin{tabular}{lcc}
\hline Symbol & Expression & Units \\
\hline$\beta$ & $I_{0} / C_{S}$ & $(\mathrm{~V} / \mathrm{s})$ \\
$\zeta$ & $I_{0} R_{L}$ & $(\mathrm{~V})$ \\
$\tau$ & $R_{L}\left(C_{L}+C_{B}\right)$ & $(\mathrm{s})$ \\
\hline
\end{tabular}

makes the linear part disappear. The derivative of (10) at the time $t=0$ is computed to get the equation of the approximating straight line, resulting in the initial slope expression of the wave segment function

$$
\left.\frac{d v_{u}}{d t}\right|_{t=0}=\beta-\frac{2 \zeta}{\tau\left(1+e^{-T / \tau}\right)} .
$$

According to the comparison between $\tau$ and $T$, the quantity $e^{-T / \tau}$ can be approximated to one. The approximating straight voltage equation is thus given by (13). Replacing $\beta, \zeta$, and $\tau$ by their respective electrical component expression, the total sensor capacitance $C_{L}+C_{B}$ can be extracted following the input values of $C_{S}$ and $I_{0}$. The parasitic capacitance $C_{B}$ is calculated according to formula (5); the parasitic permittivity $\varepsilon_{B}$ is fixed by the IDE structure board material:

$$
v_{u}(t)=\left(\beta+\frac{\zeta}{\tau}\right) t=I_{0}\left(\frac{1}{C_{S}}+\frac{1}{C_{L}+C_{B}}\right) t .
$$

3.3.2. The Linear Part of $v_{u}(t)$ Is Predominant. The exponential part of the wave segment $v_{u}(t)$ becomes negligible in comparison with the linear one $(\tau \ll T)$. Figure $7(\mathrm{~b})$ shows how the linear part is becoming predominant when the exponential part is going down. The wave segment equation is written as (14). This case occurs when the IDE sensor equivalent impedance magnitude is negligible in (6). The system response is perfectly triangular with a slope equal to the $\beta$ parameter and therefore inversely proportional to the additional capacitance $C_{S}$ :

$$
v_{u}(t)=\beta t=\frac{I_{0}}{C_{S}} t
$$

3.3.3. Both Parts of $v_{u}(t)$ Are Appearing Distinctly. This case corresponds to the general form of the segmented voltage $v_{u}(t)$ and is used to extract the fluid resistance $R_{L}$. Focusing on the $\zeta^{\prime}$ parameter, the exponential term $e^{-T / \tau}$ can be approximated to zero with an error of less than $1 \%$ when the condition $T>5 \tau$ is satisfied. The parameter $\zeta^{\prime}$ is therefore substituted by $2 \zeta$ (15), with the $\zeta$ parameter being directly linked to the fluid resistance $R_{L}$ :

$$
v_{u}(t)=\beta t+2 \zeta\left(1-e^{(-t / \tau)}\right)
$$

\section{System Design}

Working in the time domain is highly advantageous because of the immediate method to generate a square signal current and thus constantly charge and discharge the sensitive device. The system that we propose implements the modified TWV 


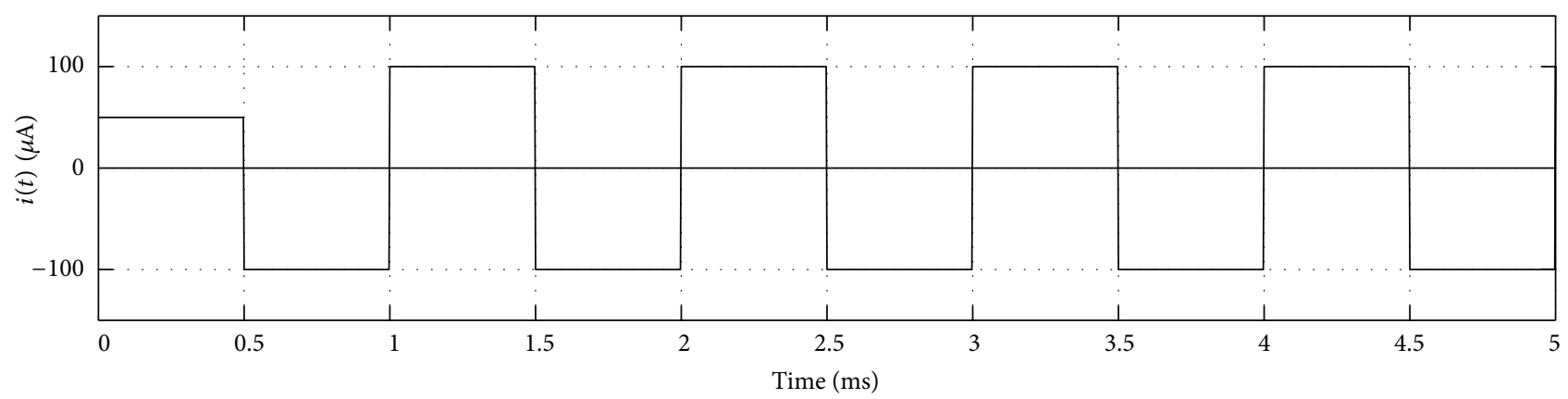

(a)

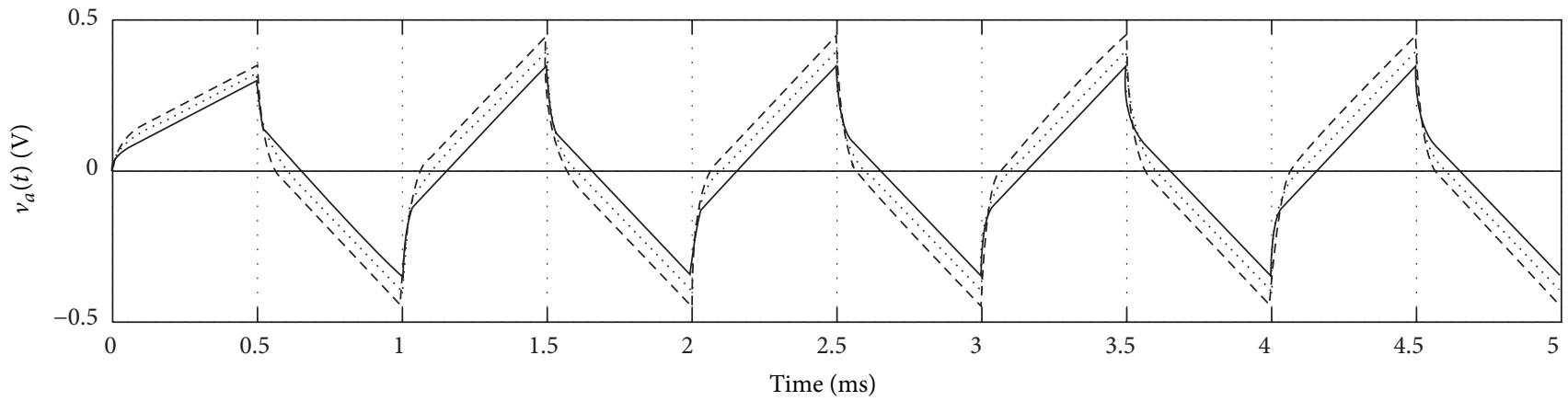

$\left[R_{L}, C_{L}, C_{S}\right]=[1000 \Omega, 5 \mathrm{nF}, 100 \mathrm{nF}]$

- - $\left[R_{L}, C_{L}, C_{s}\right]=[2000 \Omega, 5 \mathrm{nF}, 100 \mathrm{nF}]$

(b)

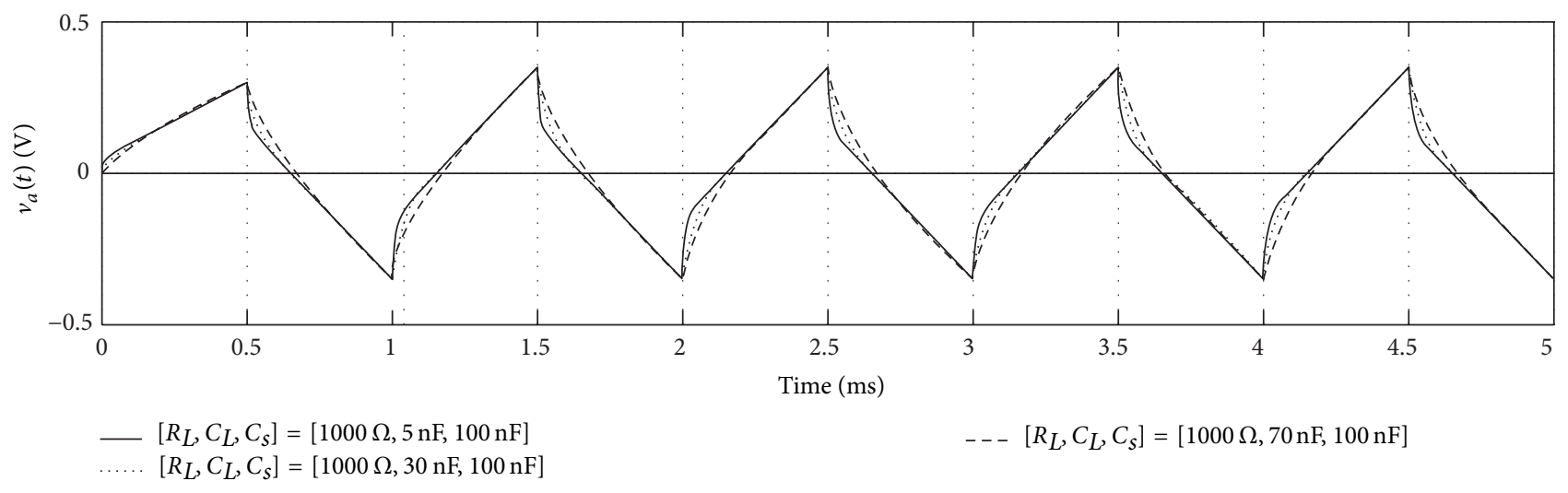

(c)

FIgURE 5: Transient square current (a) and voltage response for resistive component variation (b) and capacitive component variation (c).

technique within a current controlled oscillator (CCO) so that no variable signal source is needed to stimulate the circuit, with the oscillating wave being generated by the system itself.

4.1. Block Diagram. The system interface block diagram is given in Figure 8.

Two opposite current sources are alternatively enabled and disabled by a digital command $v_{d}(t)$ which is the system feedback control signal. The swing of the analog wave $v_{a}(t)$ is limited by the hysteresis of a Schmitt trigger. The system polarity changes each time $v_{a}(t)$ reaches the upper or the lower limit of the hysteresis voltage.

4.2. Circuit Implementation. The CCO architecture of the closed-loop system generates an oscillating signal and has been designed on CMOS advanced technologies in [10]. In the present work, as our goal is to prove an instrumentation concept and not yet to design a CMOS integrated sensor interface, we propose a similar architecture implemented with bipolar transistors to mirror the load current. The MOSFET devices act as switches to invert the circuit polarity. The analog voltage is monitored by a Schmitt NAND gate 


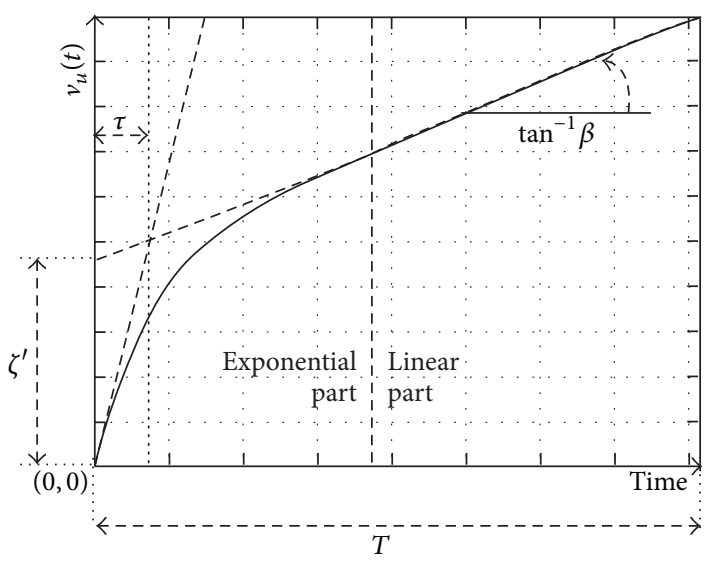

FIGURE 6: Output voltage wave segment shape predicted by (9) and (10).

which yields the feedback digital control to change the current load polarity. A high input impedance analog buffer has been added to avoid deforming the triangular wave due to the relatively low input impedance of the probing equipment.

\section{Measurements Results}

The experiments presented in this section are conducted to validate the detection methodology applied by the closedloop system proposed in the previous section. The system circuit architecture is implemented on printed circuit board (PCB) with discrete components. Such a system can also be integrated on advanced CMOS technologies. As the main goal of this research work is to prove the concept of the sensing methodology, the discrete PCB implementation is the less costly choice to validate the sensing principle. The CMOS integration is considered as a future work that will improve the system performances in terms of detection resolution, power consumption, and size reduction. This section first describes the characterization setup and the PCB platform. Preliminary tests on lumped capacitance and resistance elements are performed to illustrate the evolution of the analog output voltage. Two types of experiments are presented to extract the conductivity and permittivity of liquids. The first type of experiment characterizes the conductivity variation of $\mathrm{NaCl}$ and $\mathrm{KCl}$ solutions while the second test highlights the ability of the system to detect both the permittivity and the conductivity of glycerol-water mixtures.

5.1. Characterization Setup. The circuit architecture of Figure 9 has been implemented on a standard PCB platform (Multi Circuit Boards Ltd.) using discrete components listed in Table 3. The board includes two output connectors used to probe the analog voltage and the digital control signal. The analog buffer and the Schmitt trigger are supplied by the voltage values listed in Table 4 . The trigger hysteresis amount $V_{h}$ is fixed by the supply voltage amplitude. The serial capacitance $C_{S}$ value of the system is set to $90 \mathrm{nF}$ to fix an output frequency range starting from $100 \mathrm{~Hz}$ to $10 \mathrm{kHz}$ respecting the load current amplitude range of $10 \mu \mathrm{A}$ to
TABLE 3: PCB prototype components annotations and models.

\begin{tabular}{lc}
\hline Component type & Name \\
\hline PNP bipolar mirror & BCV62 \\
NPN bipolar mirror & BCV61 \\
CMOS switch & ADG636 \\
Schmitt NAND gate & CD4093 \\
Analog amplifier & AD8661ARZ \\
\hline
\end{tabular}

TABLE 4: System setup parameters.

\begin{tabular}{lcc}
\hline Description & Symbol & Value \\
\hline Supply voltage & $V_{\mathrm{dd}}$ & $5 \mathrm{~V}$ \\
Hysteresis voltage & $V_{h}$ & $0.9 \mathrm{~V}$ \\
Serial capacitance & $C_{S}$ & $90 \mathrm{nF}$ \\
\hline
\end{tabular}

TABLE 5: Tested lumped components calibrated values and measured fitting equation (14).

\begin{tabular}{lcccc}
\hline Signal & $R_{L}(\Omega)$ & $C_{L}(\mathrm{nF})$ & $C_{S}(\mathrm{nF})$ & \\
\hline \multirow{3}{*}{$v_{a}(t)_{11}$} & 1100 & 10 & 90 & Cal. \\
& 1140 & 10.5 & 86.5 & Meas. \\
& $3.6 \%$ & $5 \%$ & $3.9 \%$ & Err. \\
\hline \multirow{3}{*}{$v_{a}(t)_{12}$} & 1600 & 10 & 90 & Cal. \\
& 1670 & 10.7 & 86.7 & Meas. \\
& $4.2 \%$ & $7 \%$ & $3.7 \%$ & Err. \\
\hline \multirow{4}{*}{$v_{a}(t)_{13,21}$} & 2100 & 10 & 90 & Cal. \\
& 2049 & 10.7 & 87.2 & Meas. \\
& $2.4 \%$ & $7 \%$ & $3.1 \%$ & Err. \\
\hline \multirow{4}{*}{$v_{a}(t)_{22}$} & 2100 & 25 & 90 & Cal. \\
& 2152 & 24.7 & 87.9 & Meas. \\
& $2.4 \%$ & $1.2 \%$ & $2.3 \%$ & Err. \\
\hline \multirow{4}{*}{$v_{a}(t)_{23}$} & 2100 & 40 & 90 & Cal. \\
& 2187 & 38.7 & 88.7 & Meas. \\
& $4 \%$ & $3.2 \%$ & $1.4 \%$ & Err. \\
\hline
\end{tabular}

$1 \mathrm{~mA}$. The interdigitated structures are directly printed on the circuit board.

5.2. Lumped Elements Values Extraction. The analog voltage measurements have been performed using lumped components of high precision $(<0.1 \%)$ that represent the IDE sensor electrical model. The tested components are listed in Table 5; the analog voltage is probed with the Agilent Infiniium MSO8104A oscilloscope and plotted in Figure 10. These results validate the analog output wave shape variations as predicted by the analytical model of Section 3 . As the system works in closed loop, the analog wave oscillates between the two defined hysteresis levels. The values of the lumped elements have been extracted according to the compact expression of the analog voltage (15). The measured values given in Table 5 are compared to the calibrated values of the lumped components. We see that the largest error is observed for the $C_{L}$ capacitance extraction. The next section will explain in more detail the origin of this error. 


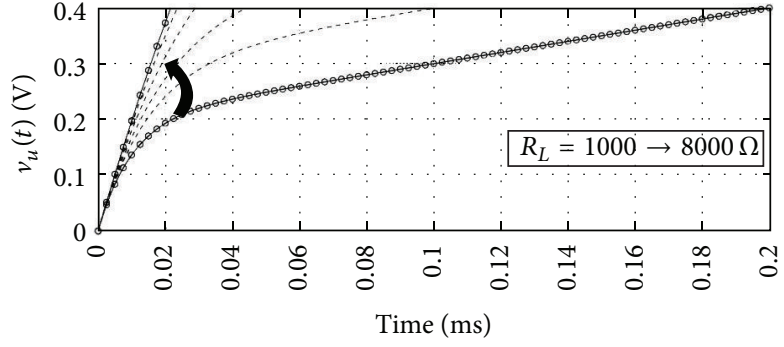

(a)

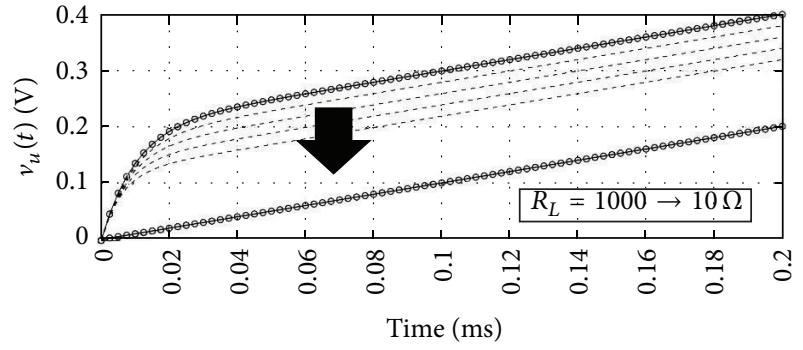

(b)

FIGURE 7: Increase and decrease effect of the exponential part of the analog voltage illustrating the cases $\tau \ll T$ (a) and $\tau \gg T$ (b).

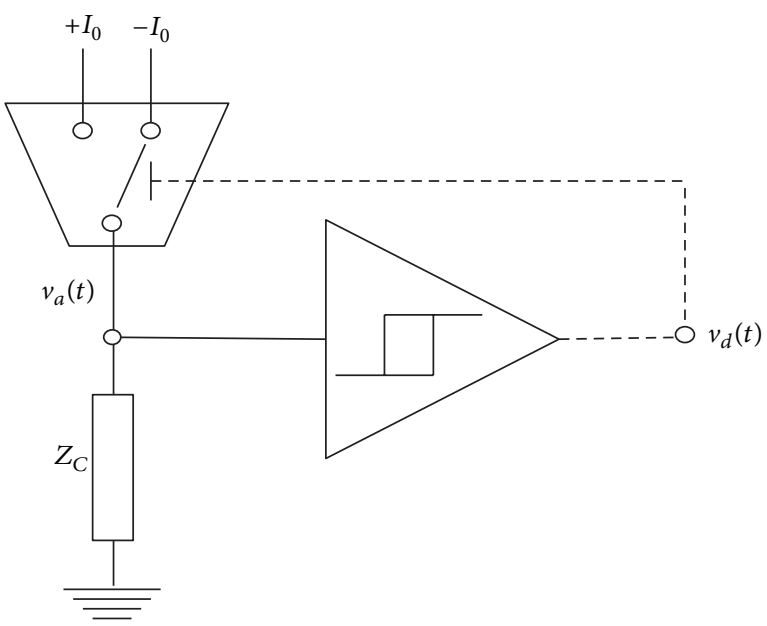

FIGURE 8: System block diagram.

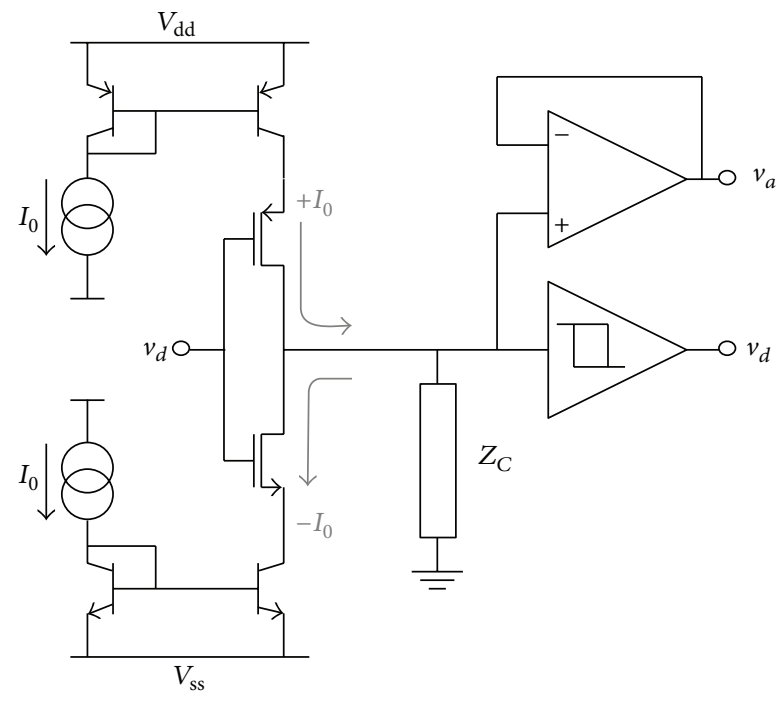

FIGURE 9: System circuit architecture.

5.3. Liquid Conductivity Measurements. The objective of the electrolyte conductivity tests is to prove the efficiency and robustness of the present sensing system. Several $\mathrm{NaCl}$ and $\mathrm{KCl}$ solutions have been prepared and calibrated using an industrial electrical conductivity meter (EUTECH CON 270, $1 \%$ full scale accuracy). The IDE sensor dimensions reported in Table 6 are chosen according to the theoretical prediction of the solutions conductivities using formula (16) in order to set a total range of liquid resistance $R_{L}$ measurable by the system, with $c_{\mathrm{XCl}}$ being the saline concentration $\left(\mathrm{mol}^{-1}\right)$ and the molar conductivities being given in Table 7 . The conductivities are then extracted using expression (5); the cell constant links the conductivity to the measured liquid resistance. The value of the cell constant gives also the IDE capacitance which is about $50 \mathrm{pF}$ for water with relative permittivity of 80 . Since this last value is out of the PCB system capacitance detection range, an additional capacitance of $5 \mathrm{nF}$ has been added in parallel with the IDE sensor:

$$
\sigma_{L}=c_{\mathrm{NaCl} \mid \mathrm{KCl}}\left(\lambda_{\mathrm{Na}^{+} \mid \mathrm{K}^{+}}+\lambda_{\mathrm{Cl}^{-}}\right) .
$$

The results plotted in Figures 11 and 12 show the progression of the liquid conductance $R_{L}^{-1}$ extracted from the system triangular output voltage. The measurements have been repeated ten times. The $x$-axis scale is the electrolyte salt concentration measured by the industrial electrical conductivity meter and expressed in $\mathrm{mol} \cdot \mathrm{L}^{-1}$ according to (16). We observe, at first sight, an excellent linearity and repeatability of the liquid measurements. The standard deviation of the measurements increases with the electrolyte concentration; a high electrolyte concentration means that the liquid resistance is small and therefore more difficult to extract. In addition, the results are compared to the expected values (continuous curves) predicted by the theoretical estimation of the cell constant; (5). The theoretical predictions and the measurements are in excellent agreement.

5.4. Liquid Permittivity Measurements. The characterization of liquid permittivity is performed by mixing two liquids of different permittivities. The two liquids have to be miscible in each other to be homogeneous and thus to create the most linear permittivity range of mixtures. We start from pure glycerol in which we add water to prepare several mixtures of different water-glycerol ratio. Knowing that the relative permittivities are about 41 for glycerol and 80 for water, the IDE sensor cell constant has to be chosen to have a significant capacitance to detect. The IDE parameters used to achieve permittivity measurements are listed in Table 8; those dimensions are chosen to increase the nominal capacitance of the 


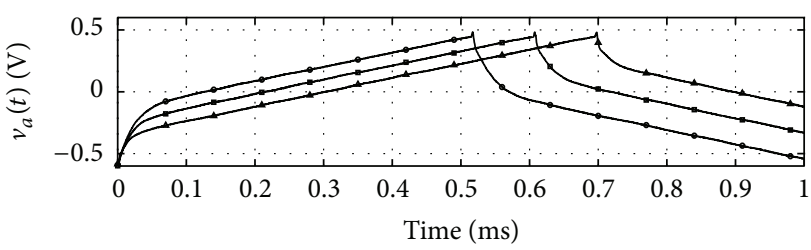

$\Delta v_{a}(t)_{11}$

$\square v_{a}(t)_{12}$

(a)

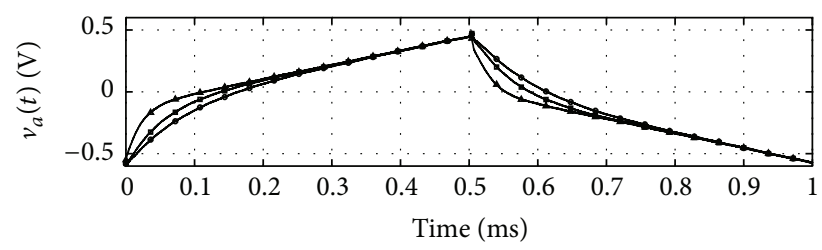

$\Delta v_{a}(t)_{21}$

○ $v_{a}(t)_{23}$

(b)

FIgURE 10: Analog voltage versus time for different values of $R_{L}(\mathrm{a})$ and $C_{L}$ (b); the legend details are reported in Table 5 .

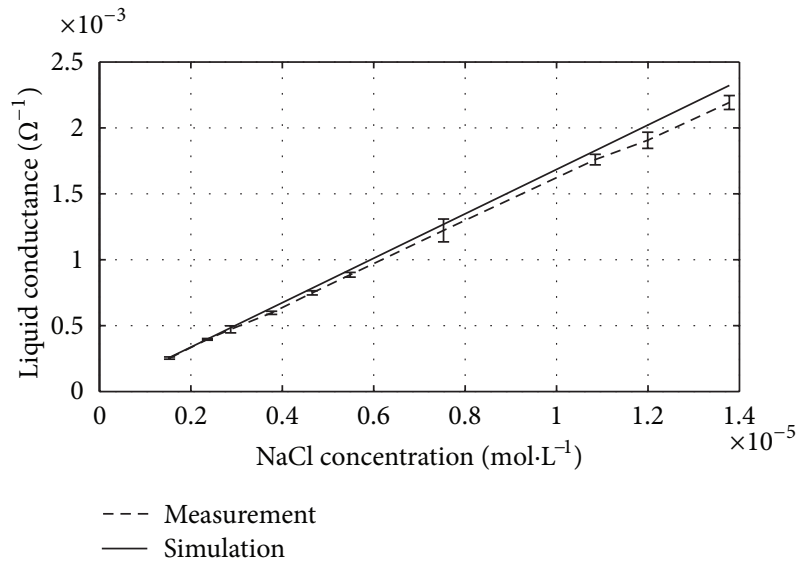

Figure 11: Experimental (with standard deviations) and theoretical evolution of the $\mathrm{NaCl}$ electrolyte conductance versus salt concentration.

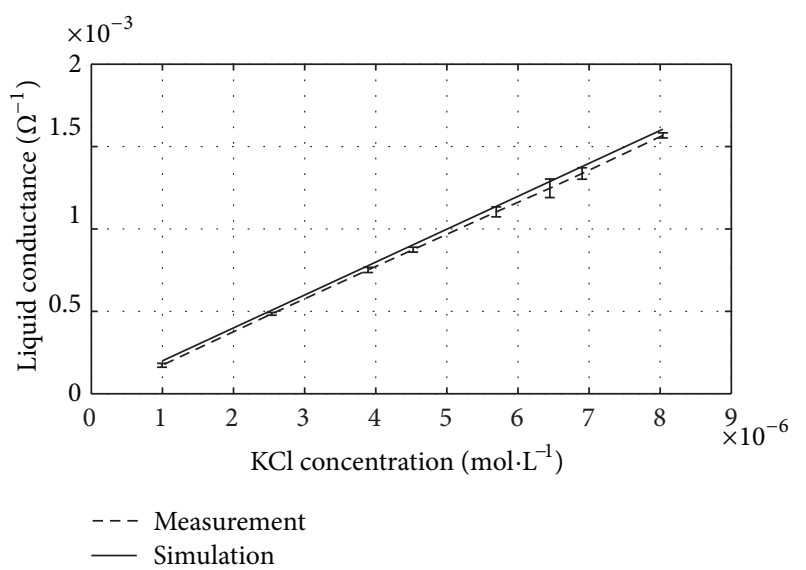

FIGURE 12: Experimental (with standard deviations) and theoretical evolution of the $\mathrm{KCl}$ electrolyte conductance versus salt concentration.

sensing element; a too small liquid capacitance $C_{L}$ involves a bad output voltage parameter extraction as explained further in Section 6. The measurements depicted in Figure 13 show the evolution of the dielectric constant versus the waterglycerol ratio of the mixture. As these two liquids have a

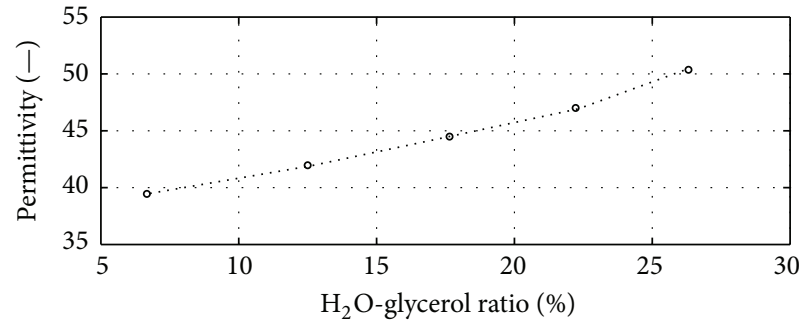

(a)

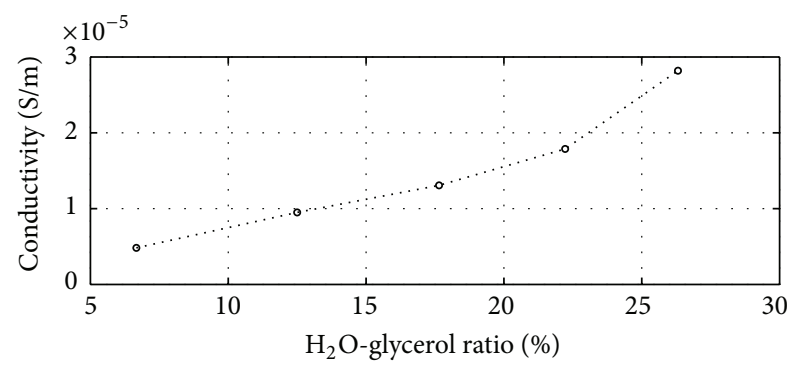

(b)

FIGURE 13: Water-glycerol mixture measurements.

TABLE 6: IDE structure parameters for saline concentration tests.

\begin{tabular}{lcc}
\hline Parameter & Value & Units \\
\hline$W_{f}$ & 500 & $(\mu \mathrm{m})$ \\
$W_{s}$ & 500 & $(\mu \mathrm{m})$ \\
$N_{f}$ & 20 & $(-)$ \\
$L_{f}$ & 11.5 & $(\mathrm{~mm})$ \\
$c_{k L}$ & 7.5 & $\left(\mathrm{~m}^{-1}\right)$ \\
$c_{k B}$ & 7.5 & $\left(\mathrm{~m}^{-1}\right)$ \\
$C_{B}$ & 3.07 & $(\mathrm{pF})$ \\
\hline
\end{tabular}

different natural conductivity, the evolution of conductivity is also shown for the mixture.

The permittivity curve versus the water-glycerol ratio was supposed to be linear due to the homogeneous prepared mixtures. The measurements shown in Figure 13 confirm this linearity. The error observed on the last point of the measured conductivity is due to the liquid resistance extraction error; the smaller the liquid resistance, the higher the impact of the extraction error. 
TABLE 7: Ionic molar conductivities.

\begin{tabular}{lc}
\hline Ion type & $\lambda\left(\mathrm{mS} \cdot \mathrm{m}^{2} \cdot \mathrm{mol}^{-1}\right)$ \\
\hline $\mathrm{Na}^{+}$ & 5.01 \\
$\mathrm{~K}^{+}$ & 7.35 \\
$\mathrm{Cl}^{-}$ & 7.63 \\
\hline
\end{tabular}

TABLE 8: IDE structure parameters for water-glycerol mixture tests.

\begin{tabular}{lcc}
\hline Parameter & Value & Units \\
\hline$W_{f}$ & 150 & $(\mu \mathrm{m})$ \\
$W_{s}$ & 150 & $(\mu \mathrm{m})$ \\
$N_{f}$ & 300 & $(-)$ \\
$L_{f}$ & 30 & $(\mathrm{~mm})$ \\
$c_{k L}$ & 0.14 & $\left(\mathrm{~m}^{-1}\right)$ \\
$c_{k B}$ & 0.14 & $\left(\mathrm{~m}^{-1}\right)$ \\
$C_{B}$ & 260 & $(\mathrm{pF})$ \\
\hline
\end{tabular}

\section{Discussion}

In every system implementation, the circuit elements are nonideal and limit the system performances. Our major concern is to insure the good quality of the analog output signal to detect capacitances and resistances on the largest measurement range; it means that the probed wave has to fit as most as possible the modeling equation (9). Therefore all parasitic impacts on the analog output voltage have to be discussed. As the system is implemented on a PCB platform by discrete components, it will be easier to highlight the system limitations. Having a good identification of these problems is important for the system implementation on any technology.

6.1. Parasitic Capacitance Effects. The most significant limitation on circuit implementations is the parasitic capacitances of the discrete components. These capacitances considerably affect the analog measurement and make the sensor capacitance more difficult to quantify, lowering the sensitivity. The parasitic effects are represented in Figure 14 with all the capacitances connected to the analog voltage node. The Schmitt trigger and the analog buffer have their own input capacitances noted $C_{i}$; the capacitances $C_{\mathrm{ds} 1}, C_{\mathrm{ds} 2}$, and $C_{\mathrm{dg}}$ are the MOSFET switches internal capacitances. Depending on where these capacitances are connected, their impact is different on the analog voltage.

The capacitances $C_{i}$ are connected to DC nodes in parallel with the sensing element; they consequently impact only the system sensitivity. The drain to source capacitances $C_{\mathrm{ds} 1}$ and $C_{\mathrm{ds} 2}$ is very small and presents a negligible impact on the measurements. The capacitance $C_{\mathrm{dg}}$ is connected between the digital command terminal and the analog voltage. Its impact deforms the analog voltage by clock feedthrough and charge injection each time the system switches [11]; the analog output response presents a voltage drop $\Delta V_{\mathrm{dp}}$ and small peaking effects compensated by oscillations which disappear after a few microseconds. The effects of the clock feedthrough and charge injection are depicted in Figure 15. The major

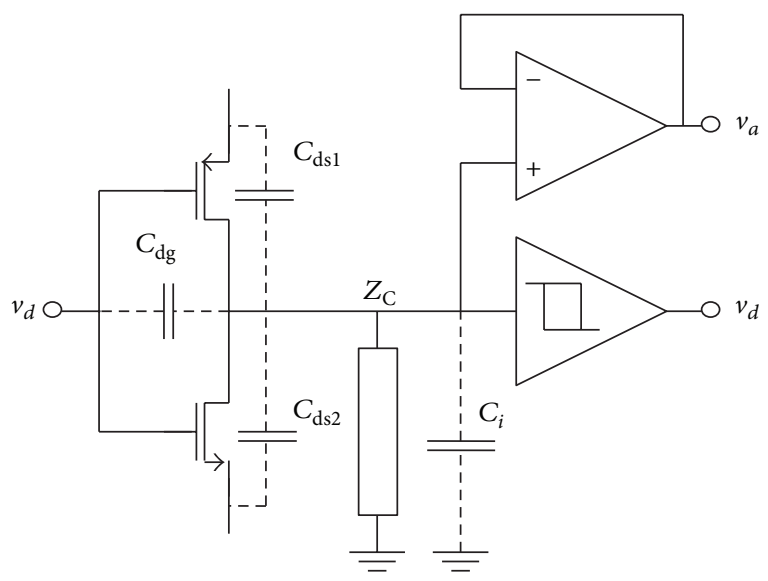

FIGURE 14: Analog voltage node parasitic capacitances.

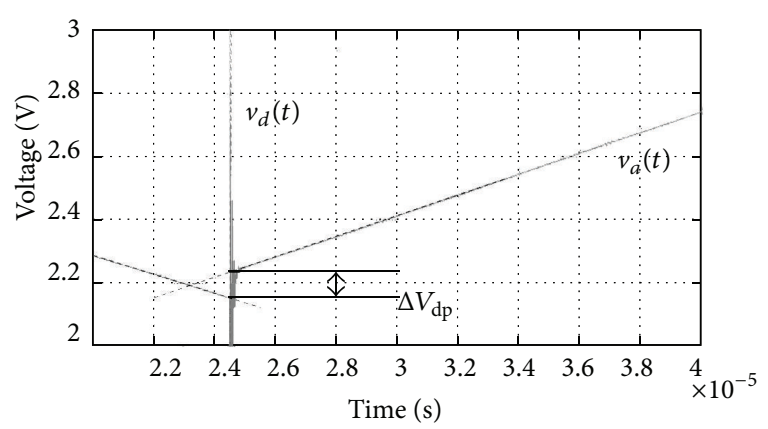

FIGURE 15: Effect of the $C_{\mathrm{dg}}$ parasitic capacitance; the clock feedthrough and the charge injection voltage drop are denoted by the black arrows.

consequence of these phenomena is bad quantification of the $C_{L}$ capacitance during the curve parameter estimation. We observed a voltage drop $\Delta V_{\mathrm{dp}}$ of $90 \mathrm{mV}$ for a tested $C_{L}$ capacitance of $200 \mathrm{pF}$. Considering the analog oscillation amplitude of $1 \mathrm{~V}$ (Figure 10), the liquid capacitance is extracted with error of up to $9 \%$. The best manner to attenuate these two parasitic effects is to integrate the circuit interface on CMOS technology. The most impacting devices that increase this effect are the CMOS switches; their surface can be drastically downscaled on integrated technology. This will reduce the coupling capacitance $C_{\mathrm{dg}}$. These effects can also be lowered by increasing the sensor nominal capacitance (i.e., increasing the finger number) to make the $C_{\mathrm{dg}}$ element small enough in comparison with the $C_{L}$ capacitance.

6.2. Circuit Bandwidth. Concerning the system frequency bandwidth, the limiting factors are the Schmitt trigger switching times and the second-order oscillations caused by the parasitic capacitances of the switches. The PCB implementation of the interface includes a 4093 NAND gate component as Schmitt trigger. Its transition time depends on the supply voltage. For the tests conditions presented in Section 5 , both the rising and falling times of the trigger are of $600 \mathrm{~ns}$. In comparison with the duration of the second-order oscillations which exceeds $1 \mu \mathrm{s}$, the bandwidth limitation is 
therefore due to the switches parasitic capacitances. For the proposed PCB implementation, we set the working frequency limit under $50 \mathrm{kHz}$; the quality of the analog output voltage is considered to be too much deteriorated beyond this limit.

6.3. Current Mirrors. The last impact on the measurements is due to the current copy performed by the bipolar transistors. Regarding the datasheet of the transistors $(B C V 6 X)$ the minimum value of the forward current gain $\beta_{f}$ is 100 ; this means that the base current error is lower than $1 \%$. The quality of the current copy is also altered by the Early effect and the mismatch between the transistors. We compensated for this error by performing a calibration which consists in measuring the mirrored amount of current injected in a reference test capacitor. In a future CMOS design, the quality of the current copy will be improved since the gate current of MOS devices is negligible in comparison with the base current of bipolar devices. In addition, the silicon integration of the circuit brings a much better matching of the mirror than discrete implementation. Nevertheless, it is also important to note that the Early effect is worse using CMOS transistors. Fortunately, this effect can be simply corrected by cascading the current mirror.

\section{Conclusions}

A modified TWV detection technique has been demonstrated to sense the $R-C$ impedance model of liquids. The theoretical result of wave segmentation includes in one compact and simple expression all the information that has to be extracted from an $R-C$ parallel equivalent sensor like IDE structures. The implementation of a closed-loop selfoscillating circuit interface makes the system autonomous and compact and requires only a few elements to be manufactured. Measurements on saline concentration have been measured with high accuracy in the $10^{-6}$ to $10^{-5} \mathrm{~mol}^{-1}$ input range and water-glycerol mixtures validated the sensing technique in respect to the correct range of permittivity (i.e., from 40 for pure glycerol to 80 for pure water). The results discussions highlighted the error sources in terms of circuit bandwidth, switching charge injection due to the parasitic capacitances, and quality of the current copy in the sensing device. The CMOS integration of the whole system would be the best solution to considerably attenuate the parasitic effects of the devices, to extend the circuit bandwidth, and also to reduce the system power consumption.

\section{Appendix}

\section{Calculation of the Response Wave Segments}

Response Wave Segmentation. Assuming that the output wave (9) is probed on a rising segment after $M$ half periods, all the hard-step functions $u(t-n T)$ for $n<M$ are elapsed and equal to 1 :

$$
\begin{aligned}
v_{a}(t, M)=\beta t & +\zeta\left(1-\frac{e^{(-t / \tau)}}{2}+\frac{3 e^{(-(t-T) / \tau)}}{2}\right) \\
& -\frac{(2 M+1) \beta T}{2}-2 \zeta \sum_{n=2}^{M}\left[(-1)^{n} e^{(-(t-n T) / \tau)}\right] .
\end{aligned}
$$

A time translation is then applied following the variable change (A.2):

$$
t^{\prime}=t-M T
$$

The expression (A.1) can be rewritten as

$$
\begin{aligned}
& v_{a}\left(t^{\prime}, M\right)= \beta t \\
&+\zeta-\zeta \frac{e^{\left(-\left(t^{\prime}+M T\right) / \tau\right)}}{2} \\
&+\frac{3 \zeta}{2} \frac{e^{\left(-\left(t^{\prime}+(M-1) T\right) / \tau\right)}}{2} \\
&-2 \zeta e^{\left(-t^{\prime} / \tau\right)} \sum_{n=2}^{M}\left[(-1)^{n} e^{(-n T / \tau)}\right]-\frac{\beta T}{2} .
\end{aligned}
$$

If we consider the square current signal as an infinite sum of hard-step functions, the expression (A.3) can be computed for $M \rightarrow \infty$. The two exponential terms of respective coefficients $-1 / 2$ and $+3 / 2$ thus disappear; a last serial progression has to be computed in the resulting equation

$$
v_{a}\left(t^{\prime}, \infty\right)=\beta t^{\prime}+\zeta-\frac{\beta T}{2}-2 \zeta e^{\left(-t^{\prime} / \tau\right)} \sum_{n=2}^{\infty}\left[(-1)^{n} e^{(-n T / \tau)}\right] .
$$

The last term of (A.4) corresponds to the well-known geometrical progression (A.5) where $s$ is the sum value, $a$ is a scale factor, and $r$ is the common ratio. The progression converges only if the common ratio $r$ modulus is smaller than unity. This is always verified in the present case because the parameters $T$ and $\tau$ are always greater than zero:

$$
s=\sum_{n=0}^{\infty} a r^{n}=\frac{a}{1-r}
$$

The final segmented expression of the output voltage wave is thus given by expression (A.6); the equation has been vertically shifted to set the starting voltage at zero:

$$
v_{u}(t)=\beta t+\frac{2 \zeta}{1+e^{(-T / \tau)}}\left(1-e^{-t / \tau}\right) .
$$

\section{Conflict of Interests}

The authors declare that there is no conflict of interests regarding the publication of this paper. 


\section{Acknowledgments}

The authors would like to thank the Wallonia Region of Belgium for funding this research work via the Skywin $H M+$ project. They also thank the staff of the WELCOME characterization platform that allowed them to perform the measurements.

\section{References}

[1] V. F. Lvovich, C. C. Liu, and M. F. Smiechowski, "Optimization and fabrication of planar interdigitated impedance sensors for highly resistive non-aqueous industrial fluids," Sensors and Actuators B, vol. 119, no. 2, pp. 490-496, 2006.

[2] R. de la Rica, C. Fernández-Sánchez, and A. Baldi, "Polysilicon interdigitated electrodes as impedimetric sensors," Electrochemistry Communications, vol. 8, no. 8, pp. 1239-1244, 2006.

[3] W. Olthuis, A. J. Sprenkels, J. G. Bomer, and P. Bergveld, "Planar interdigitated electrolyte-conductivity sensors on an insulating substrate covered with $\mathrm{Ta}_{2} \mathrm{O}_{5}$, 'Sensors and Actuators B, vol. 43, no. 1-3, pp. 211-216, 1997.

[4] J. Wu and J. P. W. Stark, "A low-cost approach for measuring electrical conductivity and relative permittivity of liquids by triangular waveform voltage at low frequencies," Measurement Science and Technology, vol. 16, no. 5, pp. 1234-1240, 2005.

[5] K.-H. Lee, J.-O. Lee, M.-J. Sohn et al., "One-chip electronic detection of DNA hybridization using precision impedancebased CMOS array sensor," Biosensors and Bioelectronics, vol. 26, no. 4, pp. 1373-1379, 2010.

[6] J. Lario-García and R. Pallàs-Areny, "Constant-phase element identification in conductivity sensors using a single square wave," Sensors and Actuators A, vol. 132, no. 1, pp. 122-128, 2006.

[7] N. André, S. Druart, P. Dupuis et al., "Dew-based wireless mini module for respiratory rate monitoring," IEEE Sensors Journal, vol. 12, no. 3, pp. 699-706, 2012.

[8] K. Bohinc, V. Kralj-Iglič, and A. Iglič, “Thickness of electrical double layer. Effect of ion size," Electrochimica Acta, vol. 46, no. 19, pp. 3033-3040, 2001.

[9] P. van Gerwen, W. Laureyn, W. Laureys et al., "Nanoscaled interdigitated electrode arrays for biochemical sensors," Sensors and Actuators B, vol. 49, no. 1-2, pp. 73-80, 1998.

[10] C. Azcona, B. Calvo, N. Medrano, S. Celma, and M. R. Valero, "A CMOS micropower voltage-to-frequency converter for portable applications," in Proceedings of the 7th Conference on Ph.D. Research in Microelectronics and Electronics (PRIME '11), vol. 26, pp. 141-144, July 2011.

[11] L. Dai and R. Harjani, "CMOS switched-op-amp-based sampleand-hold circuit," IEEE Journal of Solid-State Circuits, vol. 35, no. 1, pp. 109-113, 2000. 

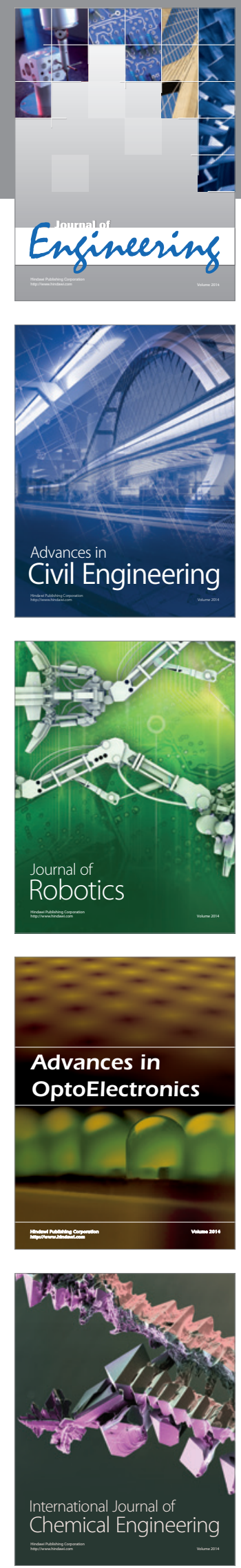

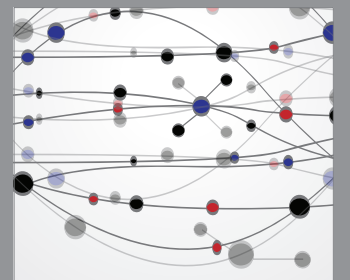

The Scientific World Journal
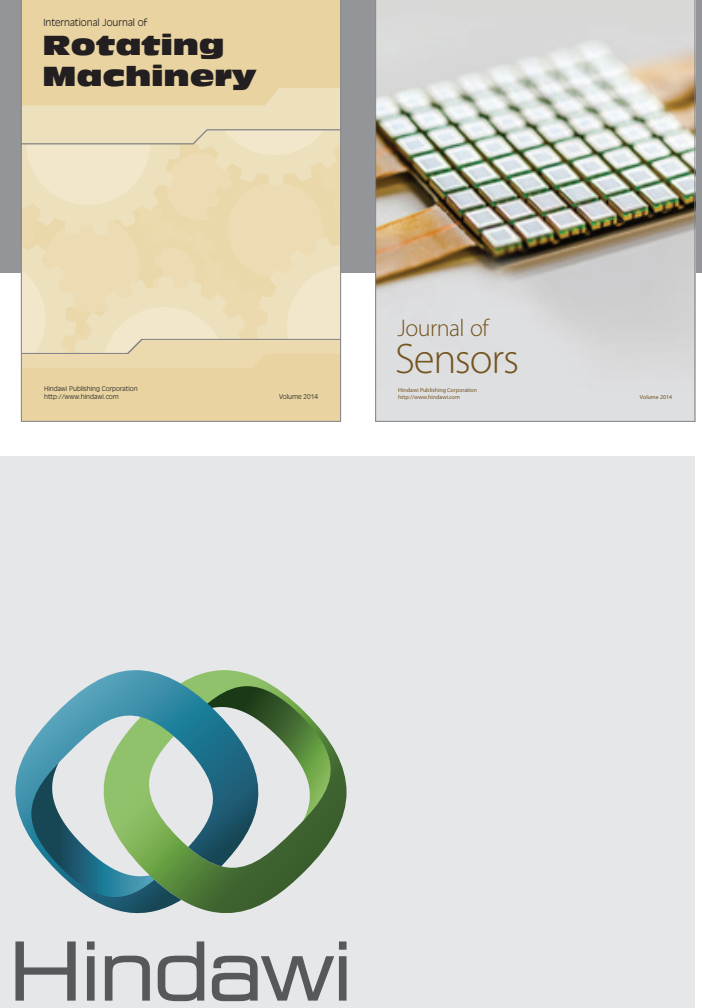

Submit your manuscripts at http://www.hindawi.com
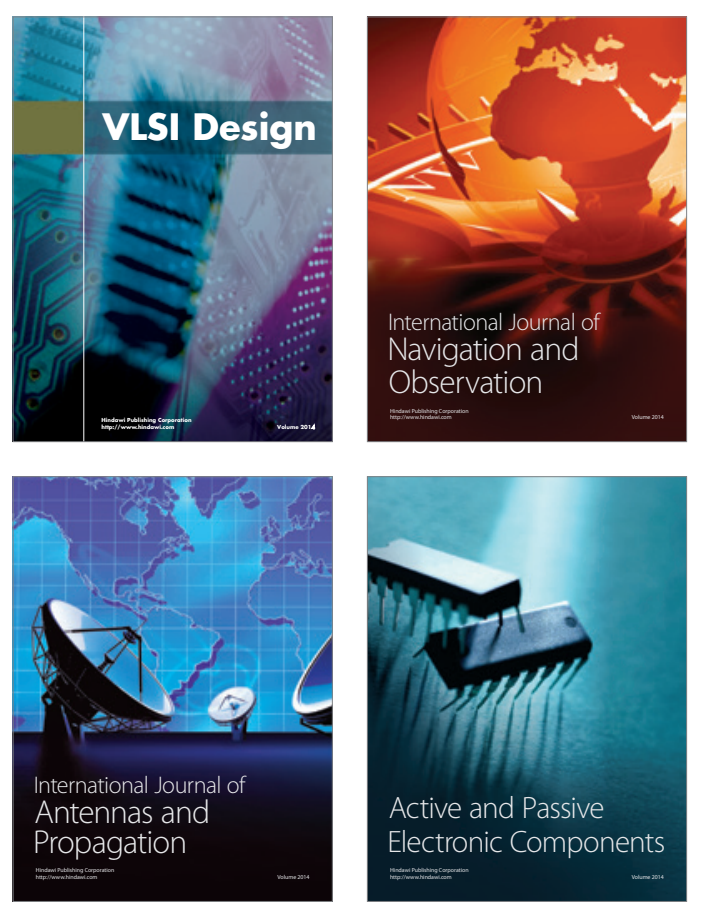
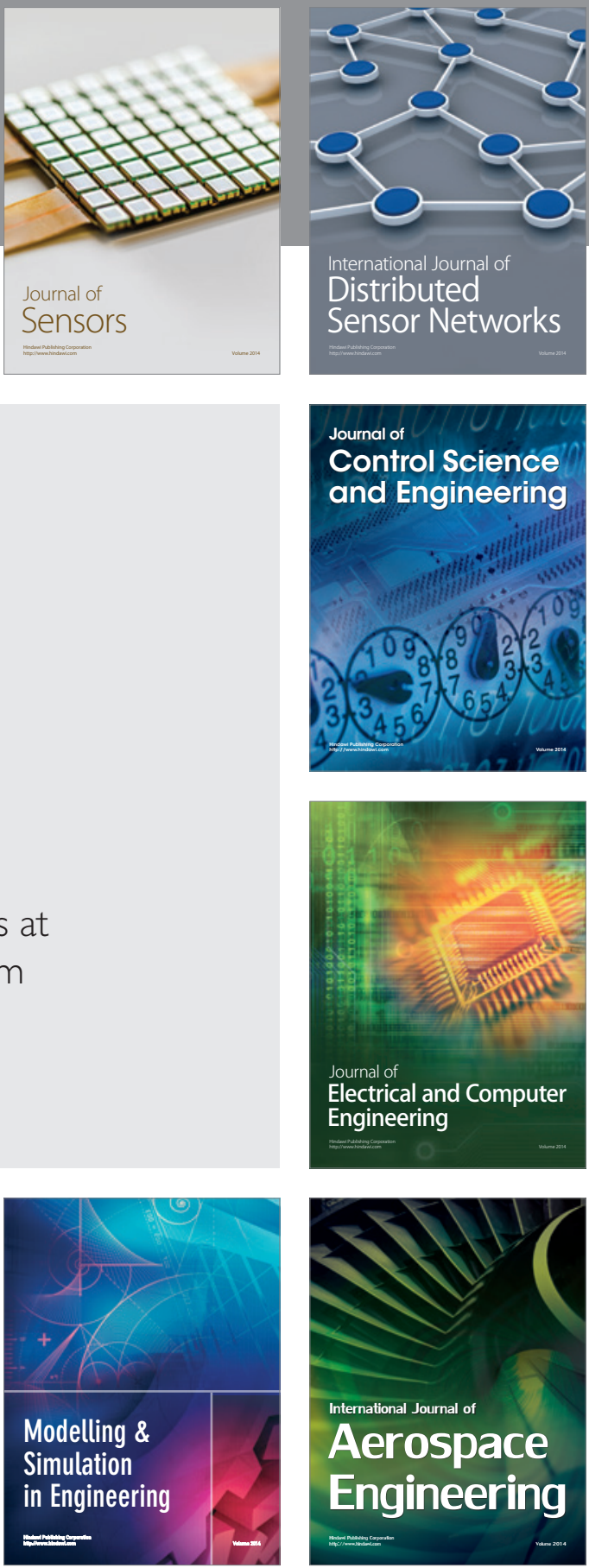

Journal of

Control Science

and Engineering
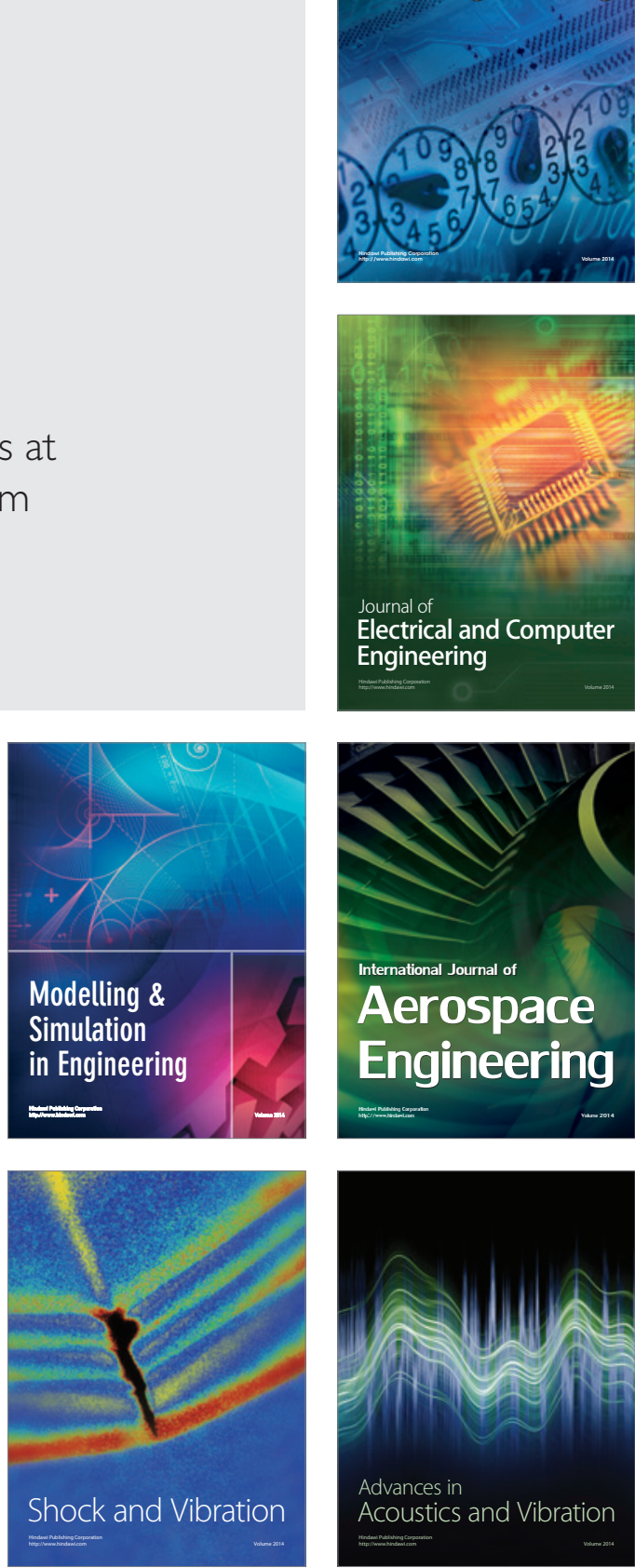